\title{
Spondylodiscitis in patients under haemodyalisis
}

\author{
Ana Maria Cervan • Juan de Dios Colmenero • \\ Alfonso Del Arco • Francisco Villanueva • \\ Enrique Guerado
}

Received: 12 November 2011 / Accepted: 13 November 2011 /Published online: 3 December 2011

(C) Springer-Verlag 2011

\begin{abstract}
Purpose The purpose of this study was to reach conclusions about the diagnosis and treatment of a series of patients with spondylodiscitis under haemodialysis treatment.

Methods We collected and studied 23 patients included in a prospective database from two neighbouring hospitals. Descriptive statistical analysis was performed.

Results The diagnosis was frequently made very late and early negative cultures were not uncommon; magnetic resonance images allowed for an early diagnosis with accurate anatomical information. Empirical antibiotic treatment with prompt surgery produced good results in patients under risk of, or having neurological problems. Surgical approaches did not differ from pyogenic or tuberculous spondylodiscitis. Age in our series was not a predictive issue.

Conclusions Whenever a patient under haemodialysis has symptoms of spinal disease, particularly back pain with fever,
\end{abstract}

A. M. Cervan $(\bowtie) \cdot$ E. Guerado

Department of Orthopaedic Surgery and Traumatology,

Hospital Costa del Sol, University of Malaga,

Carretera Cadiz-Malaga Km 187.A-7, Marbella,

29603 Malaga, Spain

e-mail: zanda_es@yahoo.es

\section{J. D. Colmenero}

Infectious Diseases Service,

Carlos Haya University Hospital,

Malaga, Spain

\author{
A. Del Arco \\ Department of Clinical Medicine, Section of Infectious Diseases, \\ Hospital Costa del Sol, University of Malaga, \\ Marbella, \\ Malaga, Spain \\ F. Villanueva \\ Department of Orthopedic Surgery and Traumatology, \\ Carlos Haya University Hospital, \\ Malaga, Spain
}

they should undergo an MRI. If diagnosis of spondylodiscitis is made prompt empirical antibiotherapy should be instituted. Blood cultures should be performed and tissue samples taken for cultures; however, culture outcome must not delay therapy. Early surgical decompression leads to good results.

\section{Introduction}

Spondylodiscitis is a very uncommon disease which is often discovered late, and early treatment appears to be very important. Patients under haemodialysis may present with spondylodiscitis as a result of bacteraemia, since the thoracic spine is the most frequent place where bacteria colonize [23]. Bacteraemia is also the first cause of morbidity and mortality in patients under haemodialysis [11]. Bacteraemia usually comes from the vascular contamination by intravenous catheters or punctures of the water-pipe [1, 7, 10, 12,13], with Staphylococcus aureus as the most frequently isolated micro-organism. Very frequently, these patients are elderly, are immunocompromised, and suffer from some other concurrent diseases [9] which presage a high morbidity and mortality rate. In hemodialysed, as in any other spondylodiscitic patient, magnetic resonance image (MRI) is the diagnostic key for prompt antibiotic treatment, as blood or sample cultures may be negative. Literature on spondylodiscitis in patients under haemodialysis is very sparse with a few patients surgically treated. The aim of this paper was to study descriptive statistics of 23 patients with spondylodiscitis under haemodialisis treated in two neighbouring hospitals.

\section{Materials and methods}

A retrospective analysis of a prospective database (from 1996 to 2010) including patients who suffered from 
spondylodiscitis while under haemodialysis treatment in two neighbouring hospitals was studied. All data were computerised (HP Doctor Hewlett-Packard Española SA 2001, License HCE 2001). Data were managed according to the Spanish Data protection law of 1999, and the study was approved by the ethics committee of our institutions. Clinical records included history, laboratory tests, diagnosis image studies, treatment, complications and final outcome.

Clinical variables included demographic data (origin, age, and gender), associated diseases, symptoms, location, and time for diagnosis. Laboratory tests included blood culture results and sample cultures. Image related diagnosis results were studied in relation to X-rays, CT-scans, and MRIs. Treatment studies included antibiotic therapy and surgical approaches. Outcome was focused on mortality and return to the former quality of life. Descriptive statistics included bivariate analyses for frequency. A chi-squared test was performed by $2 \times 2$ contingency tables (for appropriate observed frequencies, more than five, or for tables with risk factors with more than two categories). In case of $2 \times 2$ contingency tables with low observed frequency, a Fisher's exact test was carried out. For continuous covariates a Wilcoxon rank-sum test was performed (known as MannWhitney test). Due to the infrequency of cases we chose a nonparametric test instead of a classical parametric t-test based on normal distribution.

\section{Results}

We collected the records of 23 patients diagnosed with spondylodiscitis while under haemodialysis treatment. They presented the following results.

\section{Clinical}

\section{Demographic characteristics}

Eighteen patients had an urban origin (78.3\%), whereas five came from a rural area (21.7\%). Patients had an average age of 67.22 years (range, 38-85; median, 68 years; first quartile, 58.50 years; third quartile, 77 years). Ten patients $(43.5 \%)$ were younger than 67.22 years of age, and 13 $(56.5 \%)$ were older. Sixteen patients were male $(69.6 \%)$ and seven $(30.4 \%)$ female (differences for $\leq$ or $\geq 67$ years, $p=0.405)$.

\section{Associated diseases}

Nine patients had diabetes mellitus (39.1\%; differences for $\leq$ or $\geq 67$ years $p=0.669)$, seven patients had suffered a previous episode of vascular access contamination (30.4\%), seven patients a previous cardiovascular infection (30.4\%), one patient a urinary tract infection (4.3\%), one a respiratory infection $(4.3 \%)$, one patient used intravenous drugs $(4.3 \%)$, one was HIV positive (4.3\%), and 11 patients had a previous bacteraemia (47.8\%) (differences for $\leq$ or $\geq 67$ years, $p=1.00$ ).

\section{Clinical symptoms}

Obvious back pain was present in 21 patients $(91.3 \%$; differences for $\leq$ or $\geq 67$ years, $p=0.486$ ) and mild pain in one, fever in 19 patients $(82.6 \%$; differences for $\leq$ or $\geq 67$ years, $p=0.604)$, shivering in 12 cases $(52.2 \%)$, constitutional symptoms (such as weakness) appeared in 15 (65.2\%), neurological symptoms in 13 cases (56.5\%; differences for $\leq$ or $\geq 67$ years, $p=0.415$ ) with decreased sensibility in seven cases (30.4\%), and motor weakness in 12 patients $(52.2 \%)$. Back pain was localised in the cervical spine in four patients $(17.4 \%)$, in the thoracic spine in five $(21.7 \%)$, in the thoracolumbar in two $(8.7 \%)$, in the lumbar spine in $10(43.5 \%)$, and a multilevel location in two patients $(8.7 \%)$.

\section{Time for diagnosis}

The mean time between the onset of the clinical symptoms and the diagnosis of spondylodiscitis was 49.35 days (range, 5-183; median, 23; first quartile, 18.50; third quartile, $65.00)$. Eleven patients $(48.8 \%)$ had suffered clinical symptoms but without a diagnosis of spondylodiscitis for more than the median (23 days).

\section{Laboratory}

\section{Blood test}

Anaemia was present in all cases with a mean peak of haemoglobin of $10.18 \mathrm{~g} / \mathrm{dl}$ (range, 6.50-14; median, 9.9; quartile, 9.10; quartile, 11.50). The mean haematocrit was $31.36 \%$ (range, 19.00-43.00; median, 30.10; quartile, 28.80; quartile, 37.00). In all cases, leucocytes level was elevated to a mean peak of 12.002 (min 3170, median 8850, max 47860). C-reactive protein (CRP) was elevated in all patients with a mean of $87.95 \mathrm{mg} /$ dl (range, 8.30-320.00; median, 50.75; first quartile, 19.68; third quartile, 126.00).

\section{Bacteriology}

Blood cultures were positive in 12 cases $(52.2 \%)$, negative in six cases $(26.1 \%)$, and were not done in three patients $(13 \%)$. In the two remaining patients, negativity was due to a laboratory mistake in one case (4.3\%) and the other, had received antibiotic treatment for 48 hours $(4.3 \%)$. Needle aspiration biopsy (NAB) was not performed in this study group in 19 
cases $(82.6 \%)$. In those who underwent NAB a microorganisms was not identified in three cases $(13 \%)$. The microorganisms grown from either blood cultures or spine tissue (bone, intervertebral disc, paravertebral or epidural abscess) were: Staphylococcus aureus in 14 (60.90\%), Staphilococcus epidermidis in three (13\%), Streptococcus in (4.3\%), Enterococcus in one (4.3\%), and Mycobacterium TBC in one $(4.3 \%)$. Differences of bacteria in relation to age were not found (differences for $\leq$ or $\geq 67$ years, $p=0.208$ ).

Diagnosis by images tools

X-rays were performed in 13 cases (56.5\%). Computed tomography (CT-scan) was carried out in eight cases $(34.8 \%)$ and scintigraphy with labelled leucocytes in two cases (8.7\%). Magnetic resonance imaging (MRI) was the most commonly used imaging tool in this series with 21 cases $(91.3 \%)$. MRI demonstrated signs of spondylodiscitis in all 21 patients. Twenty-two cases $(95.7 \%)$ presented with a disk infection, 14 cases an epidural abscess (60.9\%; differences for $\leq$ or $\geq 67$ years, $p=0.669)$, and 13 an anterior paravertebral abscess (56.5\%); in two (8.7\%; differences for $\leq$ or $\geq 67$ years, $p=1.00$ ) out of these 13 a psoas abscess also appeared. Diagnostic imaging located the spondylodiscitis in the cervical spine in four cases $(17.4 \%)$, the thoracic in four cases $(17.4 \%)$, at the thoracolumbar junction in four $(17.4 \%)$, in the lumbar in six cases $(26.1 \%)$, and at the lumbo-sacrum in two cases (8.7\%); multilevel location was seen in three cases $(13 \%)$.

\section{Treatment}

\section{Medical treatment}

All patients were treated with intravenous wide spectrum antibiotics as soon as the micro-organism was identified. Empirical antibiotics were used in 11 $(47.82 \%)$ cases in which no micro-organism had been found. Patients received at least six weeks specific antimicrobial therapy with a mean of 73.45 days treatment (median, 53.00). Antimicrobial treatment consisted of intravenous mono or combined antibiotic therapy for a mean period of four weeks, followed by oral, mono or combined, antibiotic therapy for an additional six weeks after the clinical symptoms settled down.

\section{Operative management}

Surgery was carried out in nine cases $(39.1 \%)$. The decision to operate was made on the basis of neurological compromise, the presence of large bone destruction, progressive spinal deformity, the presence of an epidural abscess or an abscess not responding to antibiotic. In all surgically treated patients biopsy, curettage, and debridement was performed. Anterior approach with curettage, plus tricortical autograft from iliac crest and hardware fixation in one stage surgery was performed in four cases (17.4\%) (Fig. 1). Three cases $(13 \%)$ were treated by an isolated posterior approach with instrumentation for an epidural abscess and very little anterior involvement. One patient had an anterior debridement together with bone grafting followed by a second stage posterior approach, and another patient had a one stage combined approach (a posterior followed by an anterior approach). The four patients with one stage anterior debridement plus iliac crest cortical autograft and plate fixation were the oldest of our series. Seven out of the nine surgical patients already had neurological symptoms. Sciatic pain was present in five patients, hypo aesthesia in four, and a paraparesis in four patients. Neurological symptoms were relieved by surgery in five patients, one patient died, and another with paraplegia did not recover after surgery.

\section{Outcome}

Overall mean hospital stay was 42.82 days (median 34.00 , max 130). Mortality was attributed to the spondylodiscitis in three patients $(13 \%)$. One patient with concurrent diabetes and morbid obesity was operated upon in a septic situation; she had major bleeding during surgery and finally died in the intensive care unit. Another two were not operated upon; both patients died because of an acute respiratory failure. One patient had neurological sequelae and the rest returned to their former level of activity.

\section{Discussion}

Bacteraemia is a common complication in haemodialysis patients resulting from contaminated vascular access devices $[1,7,10,12,13]$. The most frequent place for bacterial colonisation is the spine [23], frequently at the thoracolumbar junction. This situation together with late diagnosis provokes a higher mortality rate $[4,15,16]$. These patients are also immunocompromised, have advanced age, and suffer very frequently from concurrent diseases such as diabetes mellitus [9]. Nonetheless, age was not an independent predictive variable in our study.

Early diagnosis is an important issue, with the suspicion of spondylodiscitis of overwhelming importance in order to perform the appropriate tests and institute antibiotic treatment. Sometimes delay in the diagnosis is too great. In our series it ranged from five to 183 days, with a median of 23 days. Eleven patients $(47.8 \%)$ waited more than 23 days without a diagnosis of 
Fig. 1 a A 78-year-old diabetic woman under haemodialysis presented with back pain for one month. X-ray showed aortic arteriosclerosis, L4-L5 discopathy, and some other degenerative changes. No other abnormalities were found. $\mathbf{b}$ MRI showed a L2-L1 spondylodiscitis. c Anterior retroperitoneal approach allowed

debridement with disk removal and vertebral bodies curettage. $\mathbf{d}$ The gap was filled with a tricortical iliac crest bone graft. Vertebral bodies were plated also anteriorly. e X-ray view after one-year follow-up (AP view). f $\mathrm{X}$-ray after one-year follow-up (lateral view)
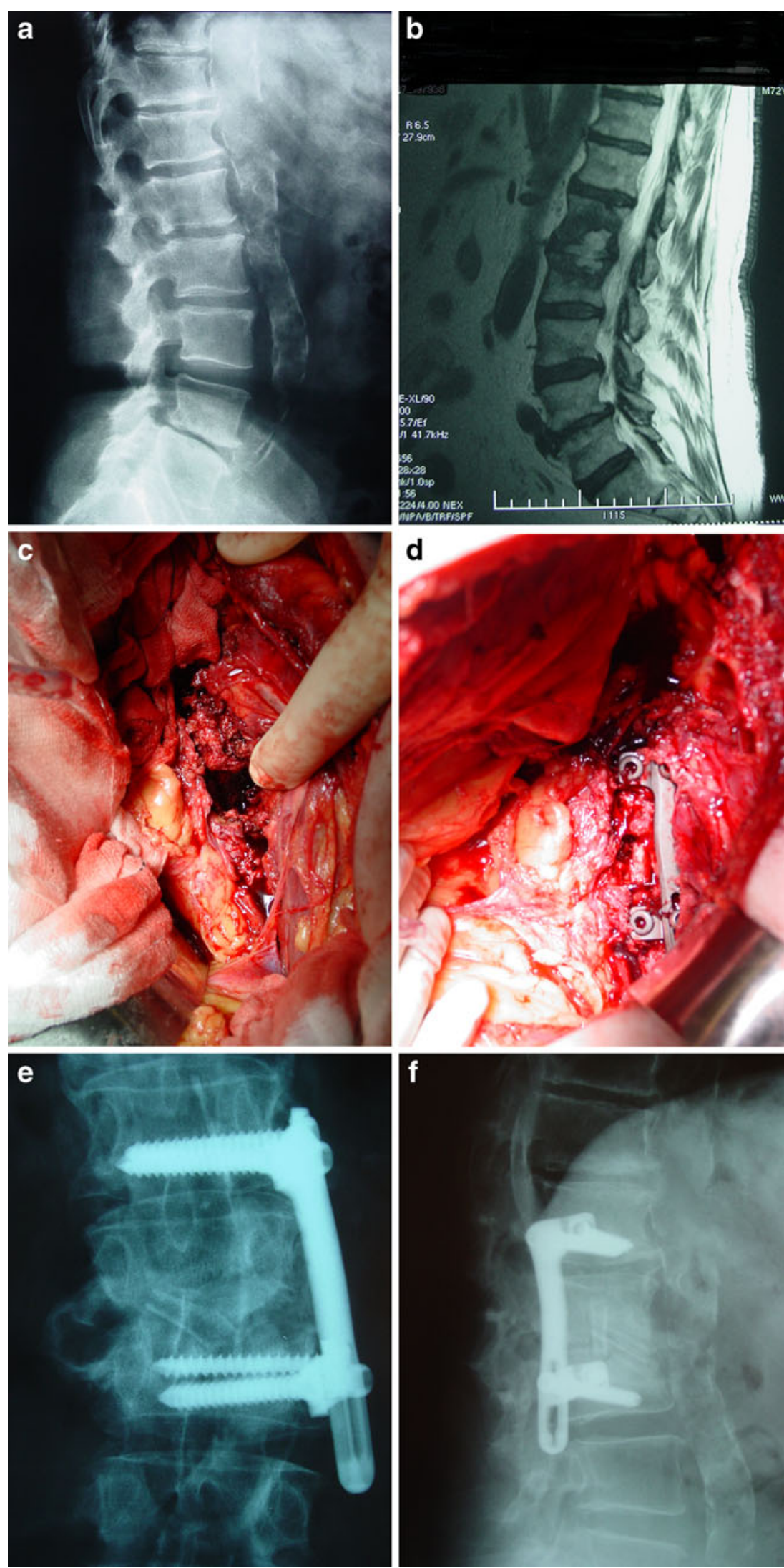
spondylodiscitis. Other authors have reported similar delays $[4,10,16,23]$. Clearly mortality could be avoided with an earlier diagnosis. Mortality rate in our series was $11 \%$ (three patients), which is similar to that published by other authors ranging from 1.5 to $15 \%[4,15,16]$.

Since early diagnosis is only achieved by MRI, this test should be performed whenever a patient under haemodialysis develops a symptom which might be suggestive of this diagnosis. The most common symptom found in our series was back pain in all but one patient $(91.3 \%)$; this is consistent with that found by other authors [24]. Fever was also very frequent with a proportion of $82 \%$ in our series. Neurological symptoms developed in more than half of our patients also because of a late diagnosis. Neurological problems progress with vertebra collapse or because of pus dissemination into the epidural space. In our series an epidural abscess was diagnosed by MRI in 14 out of 23 patients. CT-scan was performed in eight cases before elective surgery. CT-scan is very useful in identifying bone destruction, giving an idea of how much bone debridement would be required during surgery. Two patients did not undergo MRI as clinical symptoms and bone destruction clearly seen in plain Xrays and CT-scan were enough to make the diagnosis; but we currently perform an MRI study in all of our spondylodiscitic patients whether or not they are under haemodialysis. Isotope labelled leucocyte scintigraphy was carried out in two patients; this diagnostic test is no longer performed as MRI gives more information than the very non-specific scintigraphy.

MRI imaging reveals discitis, bone destruction and abscess. MRI, together with CRP, is often the only tool required for diagnosis as it is not always possible to have a biopsy and a positive culture. In 11 patients $(47.82 \%)$ we could not identify any micro-organism. Some other authors also have similar and even higher percentages $[15,21]$. However, since staphylococcus was the most common micro-organism, responsible for the infection in 14 cases $(60.9 \%)$ in our study, and also in most publications [2, 4, $5,14,19]$, antibiotic treatment should be empirically instituted early [5]. Furthermore, staphylococcal bacteriaemia should be promptly prevented if a local infection in the intravenous catheters appears, with either cloxacillin or, if a methicillin resistant staphylococcus is suspected, with vancomycin plus rifampicin.

Bibliography has demonstrated the importance of routine replacement of tunnelled cuffed haemodialysis catheters in haemodialysis patients with bacteriaemia in order to reduce infectious complications such as an epidural abscess or vertebral osteomyelitis [20]. When the bacteria is not identified we use cloxacillin alone or associated with other antibiotics. Recent studies have found no significant differences in relation to treatment outcome between patients with or without a microbiological diagnosis [25], although it is a worldwide rule that, whenever possible, therapy must be instituted with intravenous antibiotics based on cultures and bacterial sensitivity.

Surgical indications in spondylodiscitic patients are uncommon provided an early diagnosis is made. An epidural abscess was found in 14 cases $(60.9 \%)$, but only three (13\%) were surgically treated. All three were in the lumbar spine. Small abscesses can be treated with antibiotics alone if prompt diagnosis is made and early treatment applied. However, the boundary ensuring when an abscess will respond to antibiotherapy or need a surgical approach does not exist. In the three epidural abscesses treated surgically, additional pedicular instrumentation was added. Posterior instrumentation is advisable as secondary instability after laminectomy can develop, particularly if anterior bone destruction exists.

An anterior surgical approach was performed in seven out of the nine surgical cases, either as an isolated procedure or in combination with a posterior approach. We have seen that whenever an anterior approach will require large bone curettage, a preliminary posterior instrumentation will provide more stability by the time the anterior approach is performed, and allow better bone curettage and reconstruction.

Since the most frequent location for bacterial colonisation in patients under haemodialysis is the spine [23] and late diagnosis is a negative variable for higher morbidity and mortality; whenever a patient under haemodialysis develops back pain, a prompt MRI should be performed [3, 17, 18], a culture sample should be taken, and empirical antibiotic treatment should be instituted. In any case, results of blood cultures should not delay speedy antibiotic treatment [6]. A minimum of six weeks antibiotic regimen after dialysis treatment is the current choice [8, 22]. Four weeks of intravenous administration can be followed by oral therapy [16]. Surgery in cases with neurological symptoms leads to good results provided the patient is in good general condition.

Conflicts of interest The authors do not have conflicts of interest.

\section{References}

1. Almirall J, Gonzales J, Rello J, Campistol JM, Montoliu J, De la Bellasca JP, Revert L, Gatell JM (1989) Infection of hemodialysis catheters: Incidence and mechanisms. Am J Nephrol 9:454-459

2. Carrage EJ (1997) Pyogenic vertebral osteomyelitis. J Bone Joint Surg Am 79:874-880

3. Carragee EJ (1997) The clinical use of magnetic resonance imaging in pyogenic vertebral osteomyelitis. Spine 22:780-785

4. Colmenero JD, Jimenez-Mejias ME, Sanchez-Lora FJ, Reguera JM, Palomino-Nicas J, Martos F, García de las Heras J, Pachon J (1997) Pyogenic tuberculous and brucellar vertebral osteomyelitis: 
a descriptive and comparative study of 219 cases. Ann Rheum Dis 56:709-715

5. Corrah TW, Enoch DA, Aliyu SH, Lever AM (2011) Bacteraemia and subsequent vertebral osteomyelitis: a retrospective review of 125 patients. Q J Med 104:201-207

6. Danner RL, Hartman BJ (1987) Update of spinal epidural abscess. 35 cases and review of literature. Rev Infect Dis 9:265-274

7. Dobdin JF, Miller MH, Steigbigel NH (1978) Septicemia in patients on chronic hemodialysis. Ann Intern Med 88:28-33

8. Grados F, Lescure FX, Senneville E, Flippo RM, Schmit JL, Fardellone P (2007) Suggestions for managing pyogenic (non-tuberculous) discitis in adults. Joint Bone Spine 74: 133-139

9. Helewa R, Embil JM (2008) Risk factors for infectious spondylodiscitis in patients receiving hemodialysis. Infect Control Hosp Epidemiol 29:566-571

10. Helewa RM, Embil JM, Boughen CG, Cheang M, Goytan M, Zacharias JM, Trepman E (2008) Risk factors for infectious spondylodiscitis in patients receiving hemodialysis. Infec Control Hosp Epidemiol 29:567-571

11. Hoen B, Paul-Dauphin A, Hestin D (1988) EPIBACDIAL: A multicenter prospective study of risk factors for bacteriemia in chronic hemodialysis patients. J Am Soc Nephrol 9:869-876

12. Hoen B, Paul-Dauphin A, Hestin D, Mayeux D (1995) Risk factors for bacterial infections in chronic haemodialysis adult patients: A multicenter prospective survey. Nephrol Dial Transplant $10: 377-381$

13. Kessler M, Hoen B, Mayeux D, Hestin D, Fontenaille C (1993) Bacteriemia in patients on chronic haemodialysis. Nephron 64:95-100

14. Legrand E, Flipo RM, Guggengbuhl P, Masson C, Maillefert JF, Soubrier M et al, The Rheumatology Network Organization (2001) Management of nontuberculous infectious discitis. Treatments used in 110 patients admitted to 12 teaching hospitals in France. Joint Bone Spine 68:504-509
15. Malawski Sk, Lukawski S (1991) Pyogenic infection of the spine. Clin Orthop Relat Res 272:58-66

16. McHenry MC, Easley KA, Locker GA (2002) Vertebral osteomyelitis: long-term outcome for 253 patients from 7 Cleveland-area hospitals. Clin Infect Dis 34:1342-1350

17. Meyers SP, Wiener SN (1991) Diagnosis of hematogenous pyogenic vertebral osteomyelitis by using magnetic resonance imaging. Arch Intern Med 151:683-687

18. Modic MT, Feiglin DH, Piraino DW, Boumphrey F, Weinstein MA, Duchesneau PM, Rehm S (1985) Vertebral osteomyelitis assessment using MR. Radiology 157:157-166

19. Mylona E, Samarkos M, Kakalou E, Fanourgiakis P, Skoutelis A (2009) Pyogenic vertebral osteomyelitis: a systematic review of clinical characteristics. Semin Arthritis Rheum 39:10-17

20. Philipneri M, Al Aly Z (2003) Routine replacement of tunneled, cuffed, hemodialysis catheters eliminates paraspinal/vertebral infections in patients with catheter-associated bacteremia. Am J Nephrol 23:202-207

21. Punnaiah CM, Saquib H (2007) Report of 2 cases of vertebral osteomyelitis/discitis caused by Enterococcus faecalis in dialysis patients. Infect Dis Clin Pract 15:199-200

22. Roblot F, Beisnier JM, Juhel L, Vidal C, Ragot S, Bastides F, Le Moal G, Godet C, Mulleman D, Azaïs I, Becq-Giraudon B, Choutet $\mathrm{P}$ (2007) Optimal duration of antibiotic therapy in vertebral osteomyelitis. Semin Arthritis Rheum 36:269-277

23. Tsuchiya K, Yamaoka K (2004) Bacterial spondylodiscitis in the patients with hemodialysis. Spine (Phila Pa 1976) 29:2533-2537

24. Valero R, Castañeda O (2004) Clinical suspect about vertebral osteomielitis: back pain in patients with hemodyalisis by catheter related infection. Nefrologia 24:583-588

25. Yoon SH, Chung SK, Kim KJ, Kim HJ, Jin YJ, Kim HB (2010) Pyogenic vertebral osteomyelitis: identification of microorganism and laboratory markers used to predict clinical outcome. Eur Spine J 19:575-582 